\title{
Gold Nanoparticles Phase Transfer for Coating the End Face of Optical Fibers
}

\author{
Mónica Monserrat Martinez-Garcia ${ }^{1}$, Juan Luis Pichardo-Molina ${ }^{1}$, Pablo Eduardo Cardoso-Ávila ${ }^{1}$, \\ Marco Antonio Meneses-Nava ${ }^{1}$ and David Monzón-Hernández ${ }^{1}$. \\ ${ }^{1}$ Centro de Investigaciones en Óptica, León, Guanajuato, México.
}

Fiber optic sensors (FOS) have been proposed as a small sensing element with simplified optical design [1]. The FOS have gained enormous interest due to several advantages, such as real-time label-free detection, freedom from interferents in the analyte medium, simple instrumentation and low cost. Some of the earliest attempts to integrate metal nanoparticles (NPs) to optical fibers were motivated by their use as SERS probes. After that also was proposed to excite the localize surface plasmon resonance (LSPR) by the interaction of the evanescent wave of the light guided to improve the optical fiber sensors sensitivity, because the optical properties of noble metal nanoparticles are very sensitive to the refractive index changes of the surrounding medium [2]. Metal nanorods and nanoislands have been deposited on optical fiber tips by evaporation, dip and dry method, electron beam lithography, self-assembly and photodeposition, among others $[1,3,4]$. In this work, gold nanoparticles (GNPs) were prepared based on the classical Turkevich method, transferred from water to chloroform $\left(\mathrm{CHCl}_{3}\right)$, and then deposited onto the optical fiber end-face by Langmuir-Schaeffer technique [5].

First, GNPs synthesis was done by boiling $40 \mathrm{~mL}$ of $3 \mathrm{mM}$ sodium citrate solution under magnetic stirring to which $40 \mu \mathrm{L}$ of $0.25 \mathrm{M}$ chloroauric acid solution was added; the solution turned transparent, then purple and finally changes to ruby red in color [6]. After that, phase transfer was made by mixing $5 \mathrm{~mL}$ of GNPs solution with $25 \mu \mathrm{L}$ of PEG-SH $(2 \mathrm{mg} / \mathrm{mL}$, O-[2-(3Mercaptopropionylamino)ethyl]-O'-methylpolyethylene glycol, Sigma-Aldrich) under vigorous magnetic stirring. In a separate glass flask, $50 \mu \mathrm{L}$ of 1-Dodecanethiol (DDT, Sigma-Aldrich) were dissolved in $5 \mathrm{~mL}$ of chloroform. After stirring for $10 \mathrm{~min}$, the $\mathrm{DDT}-\mathrm{CHCl}_{3}$ solution was added to the GNPS solution coated with PEG-SH and left to react for $20 \mathrm{~min}$; then $80 \mu \mathrm{L}$ of $3 \mathrm{M}$ hydrochloric acid were added to the solution under magnetic stirring for two hours, the flask bottom become ruby red in color, indicating that the GNPs were transferred to the chloroform phase. The two phases were divided by a separating-funnel, and then GNPs were washed twice by centrifugation at 7300g. The GNPs UV-Vis extinction spectra were acquired using a portable spectrometer (Stellarnet, EPP2000) before and after the phase transfer. The extinction plasmon band exhibited a red-shift from 518.5 to $535 \mathrm{~nm}$, due to the change in the media refractive index where GNPs were suspended (see Figure 1A). For the scanning electron microscopy (SEM) analysis, GNPs in water and in chloroform were dropped onto an aluminized glass substrate and dried at room temperature; the SEM micrographs (JEOL, JSM-7800F) showed the mean sizes of $15 \pm 3 \mathrm{~nm}$ for the GNPs in water and $14 \pm 1 \mathrm{~nm}$ for GNPs after the phase transfer (see, figures $1 \mathrm{~B}$ and 1C).

A mono-mode optical fiber with a $8.2 / 125 \mu \mathrm{m}$ core/cladding diameter (SNS-28, Corning) was prepared for the GNPs deposit: first the polymer coating was mechanically removed; the cladding surface was cleaned with acetone and the fiber tip cut with a cleaver. Afterward, it was treated with a plasma cleaner (Femto, Diener electronic) for 10min under oxygen atmosphere. Then, a glass flask $(5 \mathrm{ml})$ was filled with water, and a GNPs self-assembled monolayer at the air/water interface was prepared by dropping carefully $5 \mu \mathrm{L}$ of GNPs in chloroform on the top of the water surface; this hydrophobic GNPs film was carefully touched with the optical fiber tip to deposit the GNPs on it. The optical fiber was rinsed plenty with acetone to remove the surfactant excess. The SEM microscopy images at low magnification (700x) revealed that GNPs were deposited over all the fiber's end-face, but a more dense monolayer was deposited at the center of the fiber(Figure 1D). 
Figures $1 \mathrm{E}$ and $1 \mathrm{~F}$ show a higher magnification $(50000 \mathrm{x}$ and $100000 \mathrm{x}$ respectively) of the GNPs monolayer, and it can be seen that the GNPs did not show aggregates or multilayer formation.

Here, we proposed an easy method with high simplicity to deposit GNPs on the optical fibers endface surface, but our method can be applied also to deposit NPs along of the core of the optical fiber and can be expanded to many other small anisotropic NPs.

References:

[1] J. Chen et al. Sensors, 15 (2015), p. 12205.

[2] X. Zheng et al., Langmuir, 24 (2008),p 4394.

[3] N. Sharifi et al., Materials Chemistry and Physics, 113(2009), p 63.

[4] J.G. Ortega-Mendoza et al., Sensors, 14 (2014), p. 18701.

[5] A. Serrano-Montes et al. Langmuir, 31 (2015), p. 9205.

[6] J. Kimling et al., The Journal of Physical Chemistry B, 110 (2006), p. 15700.

[7] The authors acknowledge M.C. Albor-Cortés, M. Olmos-Morales, J.L. Flores-Arias and B.

Reyes-Ramirez for their technical support.

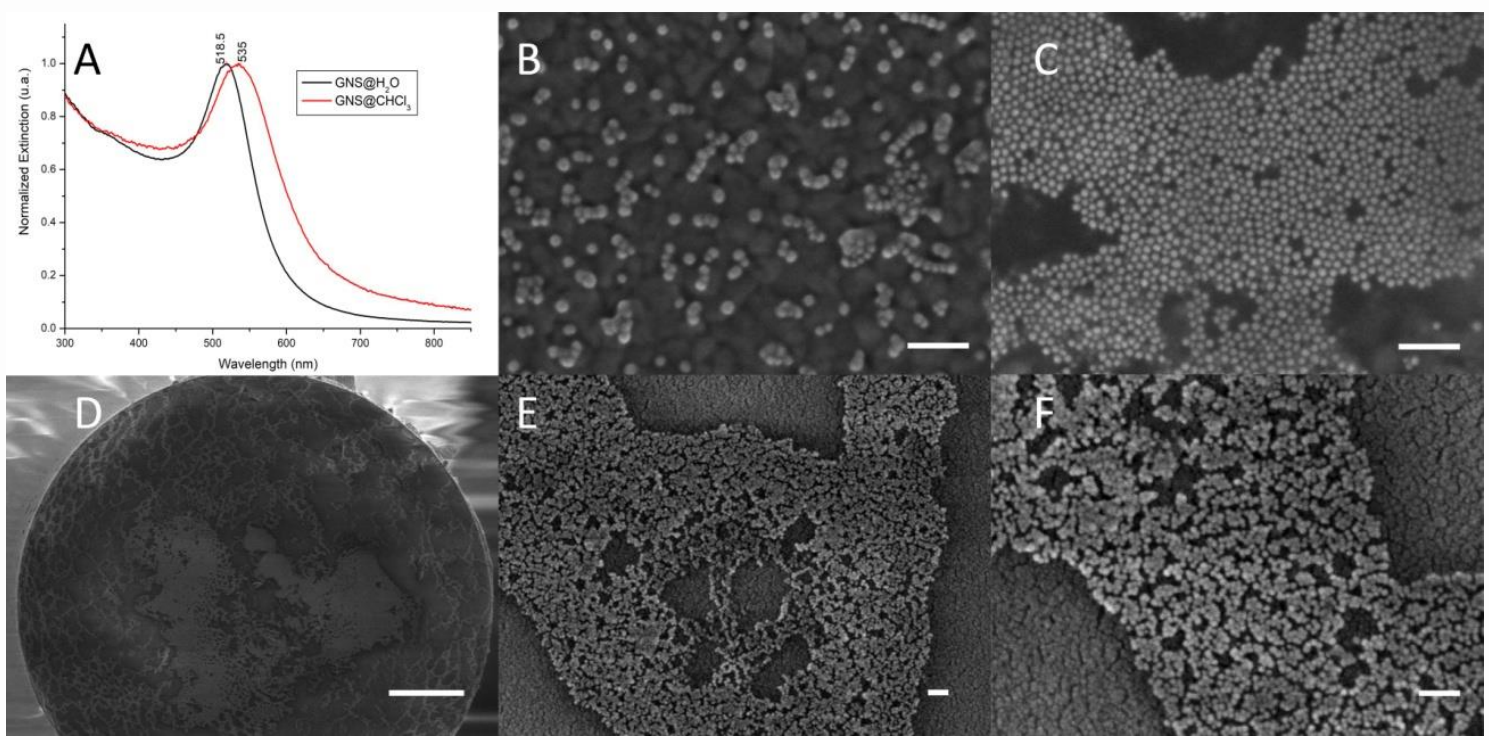

Figure 1. Normalized UV-Vis extinction spectra of the $\mathrm{GNPs} / \mathrm{H}_{2} \mathrm{O}$ and $\mathrm{GNPs} / \mathrm{CHCl}_{3}(\mathrm{Panel} \mathrm{A})$. SEM microscopies of the GNPs $/ \mathrm{H}_{2} \mathrm{O}$ and $\mathrm{GNPs} / \mathrm{CHCl}_{3}$ deposited onto aluminized glass substrates (B and $\mathrm{C}$ respectively). SEM images of the $\mathrm{GNPs} / \mathrm{CHCl}_{3}$ deposited over the optical fiber tip at different magnifications (D to F). Scale bar is $20 \mu \mathrm{m}$ in panel D, and $100 \mathrm{~nm}$ in panels B, C, E and F. 